\title{
Computational study of stacked complexes of aliphatic and aromatic species
}

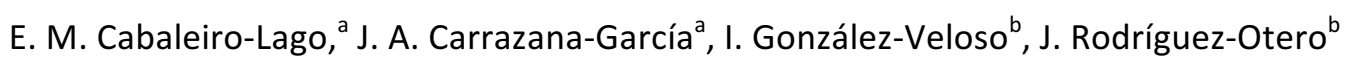

\footnotetext{
${ }^{a}$ Facultade de Ciencias (Dpto. de Química Física), Universidade de Santiago de Compostela, Campus de Lugo. Avda. Alfonso X El Sabio s/n 27002 Lugo, Galicia (Spain).

${ }^{b}$ Facultade de Química (Dpto. de Química Física), Universidade de Santiago de Compostela, 15782 Santiago de Compostela, Galicia (Spain).
}

\begin{abstract}
The interaction between extended aromatic species increases with system size at greater rate than interactions between aliphatic analogues or mixed aliphatic-aromatic ones. A series of complexes formed by benzene and cyclohexane with several acenes and their saturated derivatives has been computationally studied by using the TPSSD3BJ/def2-TZVPP level of calculation. Considering prototypical structures, the equilibrium distances and interaction energies of the complexes have been obtained. The results are used to check on the characteristics of similar $\pi$-extended $\pi, \sigma$ extended $\pi, \pi$-extended $\sigma$ and $\sigma$-extended $\sigma$ complexes.
\end{abstract}

\section{Keywords:}

Intermolecular interactions; $\pi-\pi$ interactions; cyclic systems; PAHs 


\section{Introduction}

Interactions between aromatic species are crucial in materials science and, more specifically, in the behaviour of extended $\pi$ systems as graphene, fullerenes and nanotubes.[1] The interactions established by aromatic species usually correspond to $\mathrm{XH}-\pi$, ion- $\pi$ or $\pi-\pi$ interactions, which seem to show specific characteristics that differentiate them from similar interactions with aliphatic species.[2]

Thus, $\pi$ stacking interactions have been long recognized as one of the key motifs contributing to the stability of aromatic systems. Though it has been commonplace to consider $\pi$-stacking as a special kind of interaction, this has been recently questioned by different authors. $[3,4]$ In fact, the interaction in cyclohexane dimer is quite similar in magnitude to that of benzene dimer, and the mixed cyclohexane-benzene dimer is even more stable.[5, 6] In consequence, there seems to be nothing special about $\pi-\pi$ stacking, and the term should be considered as a mere geometry descriptor regarding the relative orientation of the monomers constituting a complex.

Though the stability of aromatic and aliphatic stacked structures is similar, the behaviour becomes different as the size of the interacting species increases. The stability grows faster in homodimers of aromatic species than in their aliphatic or mixed aromatic-aliphatic counterparts. This is mainly because dispersion, the main stabilizing contribution in these complexes, grows faster in aromatic systems than in aliphatic ones as the size of the system increases. The main factor favouring aromatic dimers over aliphatic ones as the size of the system increases is related to the nature of the aromatic systems. Changes in molecular properties due to larger electron delocalization as the aromatic system grows lead to an increase in the dispersion contribution to the stability, absent in aliphatic dimers. Besides, an extra stabilization is observed in $\pi-\pi$ dimers related to the softening of the repulsive wall that allows shorter intermolecular distances. $[4,7,8]$

In this work, preliminary results are presented on the characteristics of the interaction between benzene and cyclohexane with a series of acenes and their saturated counterparts of growing size. Figure 1 shows the systems studied in this work, comprising benzene, anthracene and pentacene, as well as their perhydrogenated derivatives. 


\section{Computational Details}

Dimers formed by benzene and cyclohexane with the species shown in Figure 1 are employed to study the effect of enlarging the system on $\pi-\pi, \sigma-\pi, \pi-\sigma$, and $\sigma-\sigma$ stacking interactions. Interaction energy curves for each of the dimers have been obtained, the intermolecular distance $\mathrm{R}$ between molecules being changed along a series of values while keeping the monomer geometries frozen at the values obtained for the isolated molecules at the TPSS-D3BJ/def2-TZVPP level. The resolution of the identity approach is used in these calculations employing the def2-TZVPP auxiliary basis set. These calculations were performed with Turbomole 6.3.[9] In order to gain more insight about the characteristics of the interaction in the dimers, the Non-Covalent Interaction Index $(\mathrm{NCl})$ has been employed.[10,11] $\mathrm{NCl}$ is an index based on the analysis of the reduced density gradient that can be employed to visualize both favourable and unfavourable interactions. These interactions can be graphically displayed as a plot of the product of the sign of the second eigenvalue of the hessian of the density times the density $\left(\operatorname{sign}\left(\lambda_{2}\right) \rho\right)$, mapped onto an isosurface of reduced density gradient.
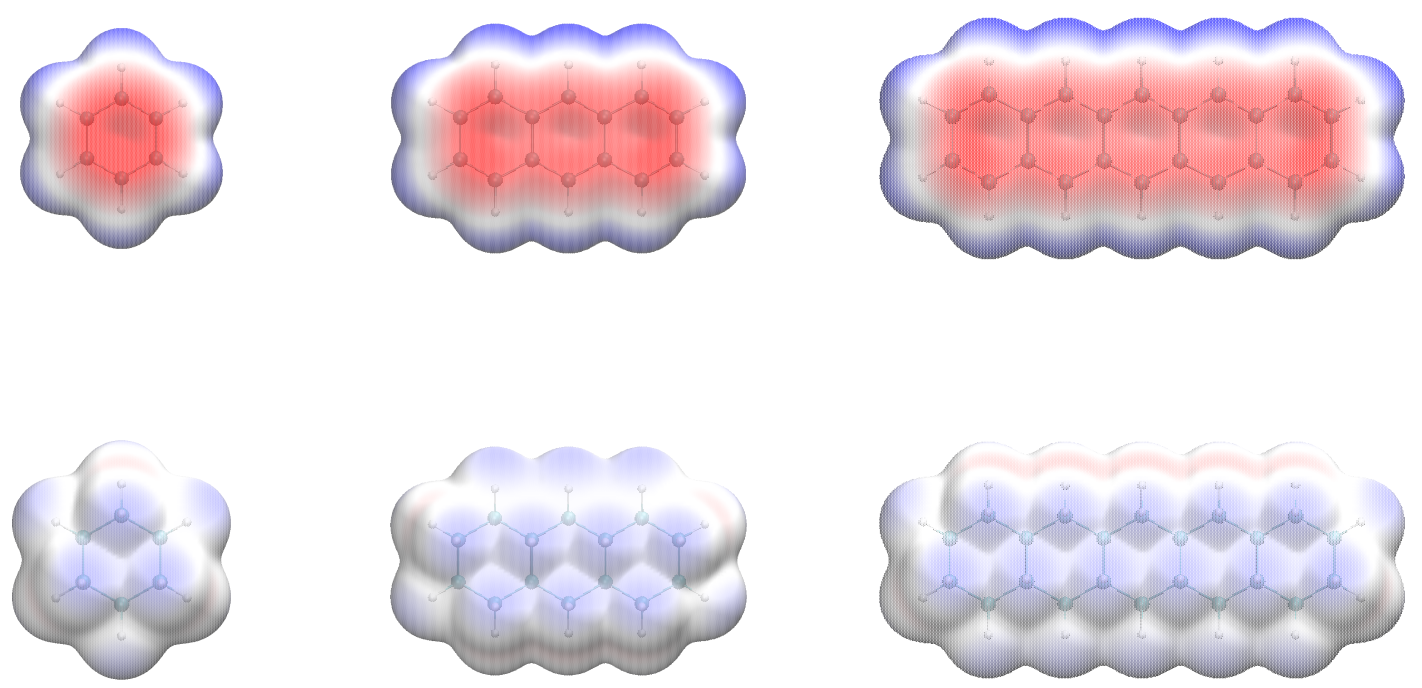

Figure 1. Molecular Electrostatic Potentials (MEP) at the TPSS-D3BJ/def2-TZVPP level. MEP mapped onto an isodensity surface of $0.001 \mathrm{a.u}$. Colour scale runs from -0.020 a.u. (red) to 0.020 a.u (blue). White is 0.0 . 


\section{Results}

Figure 1 shows the Molecular Electrostatic Potentials (MEPs) of the molecules considered in this work, showing the large differences between aromatic and aliphatic species. As it is widely known, the main characteristics on the MEP of unsubstituted aromatic species is the clearly negative region above and below the ring related to the aromatic cloud. The MEP over the central ring becomes less negative as the aromatic system is extended, becoming neutral for graphene. On the other hand, aliphatic species show a slightly positive MEP associated to the hydrogen atoms, which barely changes with system size. MEPs can be informative on some characteristics of the interaction, though they must be considered with care, since the main stabilizing factor in stacked structures comes from dispersion.

Figure 2 summarizes the dimers considered in this work. The structures are built by locating cyclohexane and benzene on top of the central ring of the other species forming the dimer. In complexes containing aromatic species one of the molecules is displaced along a $\mathrm{C}-\mathrm{C}$ bond because these displaced structures are more stable, leading to a graphite-like arrangement in $\pi-\pi$ complexes, and to $\mathrm{CH}-\pi$ interactions in mixed complexes.[8] Thus, only $\sigma-\sigma$ complexes are fully stacked.

The Non-Covalent interaction Index ( $\mathrm{NCl}$ ) allows to display in a graphic way intermolecular interactions based on the characteristics of the charge density. These interactions can be visualized in the 3D plots in Figure 2 for the dimers studied at their equilibrium distances. It can be observed that in mixed dimers the plots reveal quite localized interaction surfaces that correspond to the presence of favourable $\mathrm{CH}-\pi$ interactions. In the case of the $\sigma-\sigma$ complexes, the favourable interactions are related to contacts among the $\mathrm{CH}$ groups of the molecules, revealed in Figure 2 as small surfaces. Finally, in $\pi-\pi$ complexes, the interacting surface is more extended.

When passing from $\mathrm{C}_{6}$-containing complexes to larger ones there is a clear size effect leading to more extended interacting surfaces. While there is one $\mathrm{CH}-\pi$ contact in benzene-cyclohexane dimer, two are observed in larger $\sigma-\pi$ systems, suggesting that the stability must increase. A similar extension is observed in the rest of the systems; the number of favourable contacts increases and the interacting surfaces become larger. However, when going from $C_{14}$ to $C_{22}$ complexes the changes are more subtle because the new atoms are located further away from benzene and cyclohexane, leading to $\mathrm{NCl}$ surfaces qualitatively similar to those obtained for $\mathrm{C}_{14}$ complexes. 

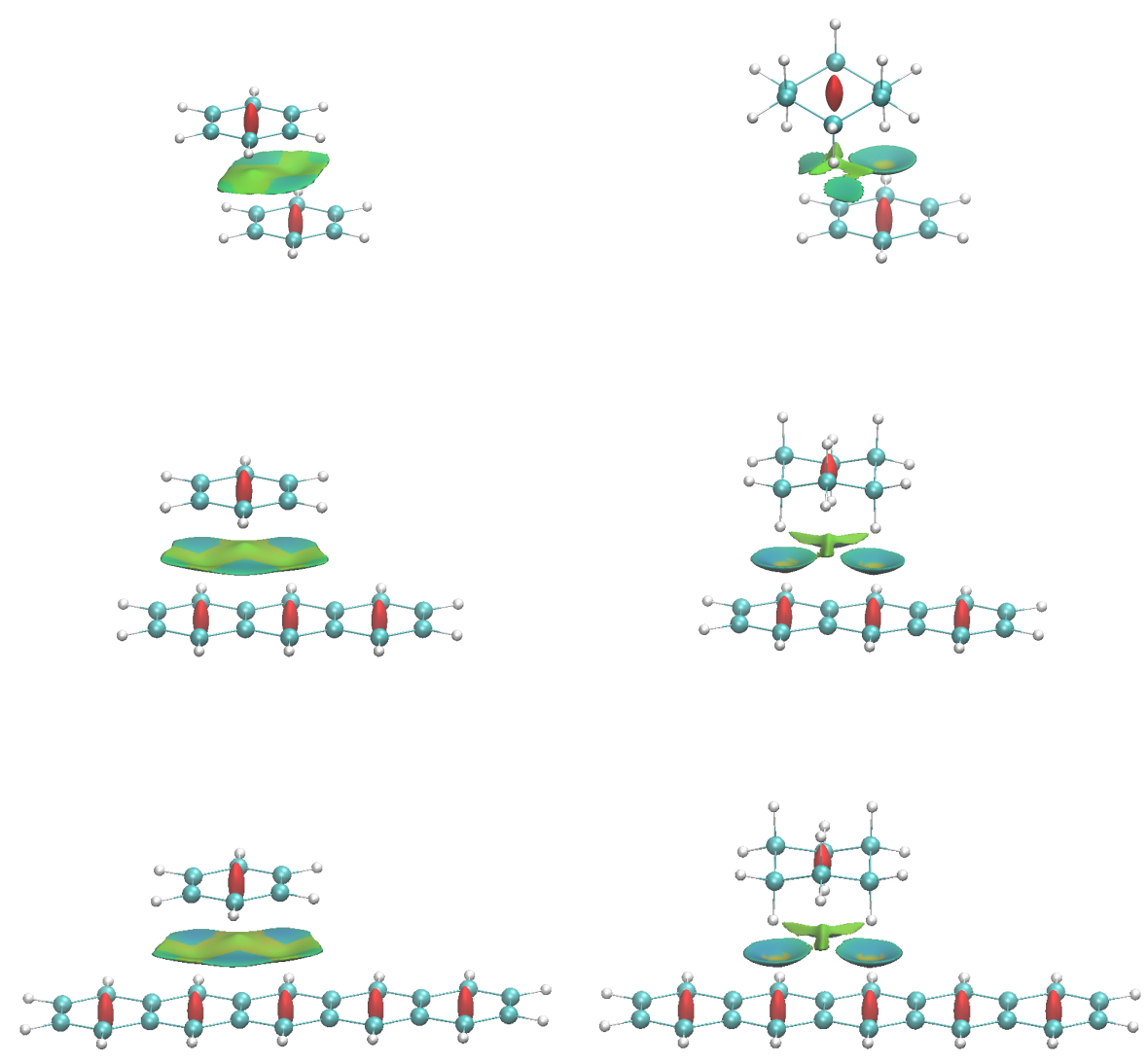

$\pi-\pi$ $\sigma-\pi$
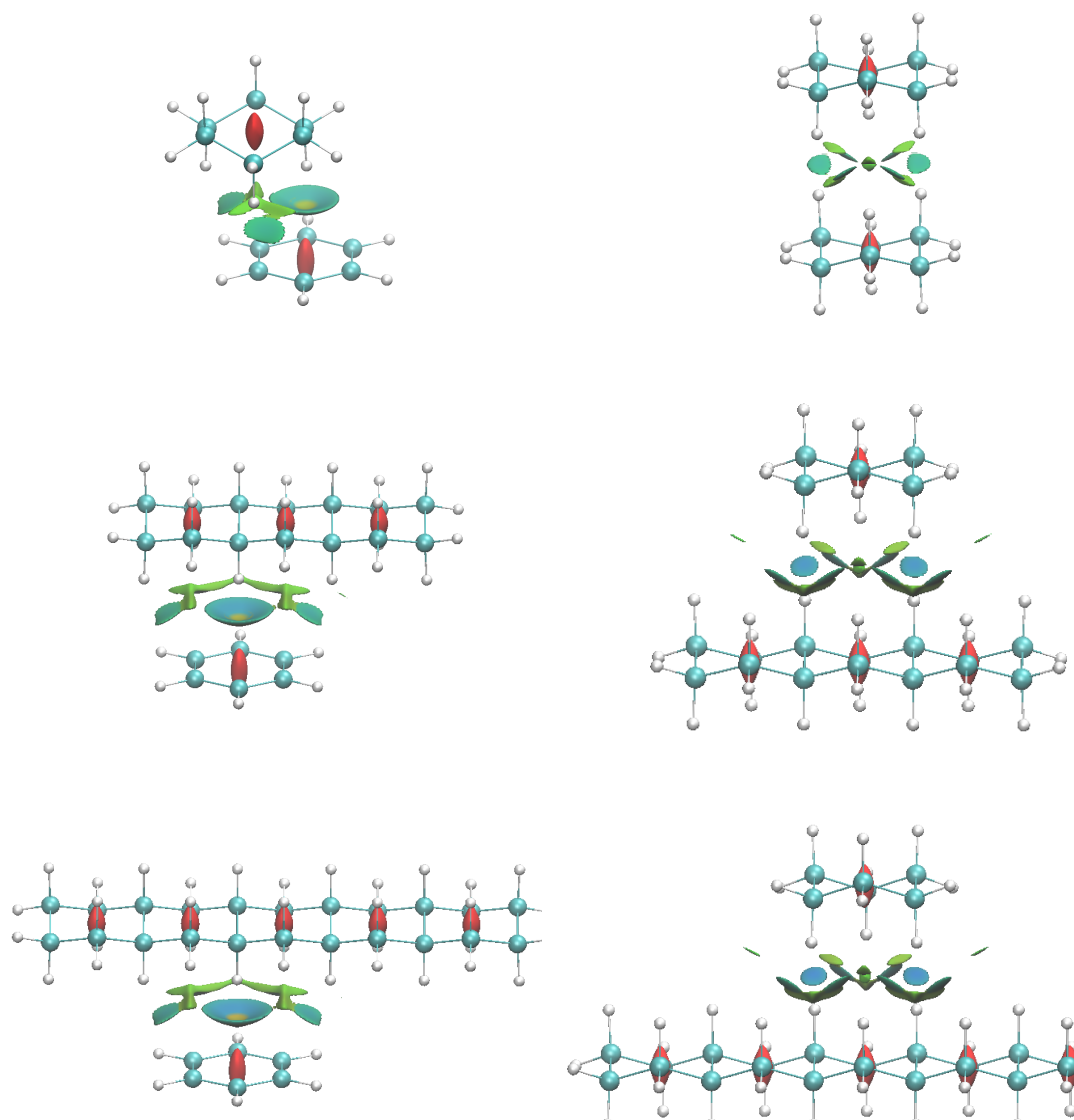

$\pi-\sigma$

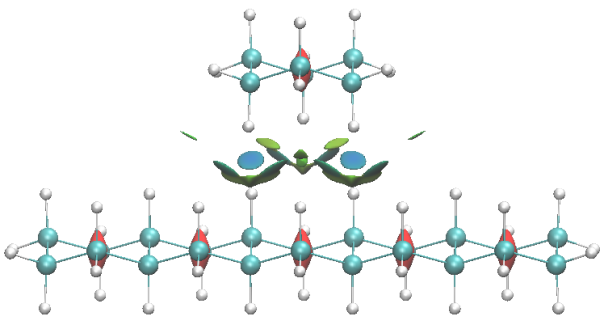

$\sigma-\sigma$

Figure 2. $\mathrm{NCl}$ plots at the minima of the complexes studied. The product of the density times the sign of the second eigenvalue of its Hessian is mapped onto an isosurface of reduced density gradient with value 0.5 a.u. The colour scale goes from -0.01 a.u. (blue) to +0.01 a.u. (red). 


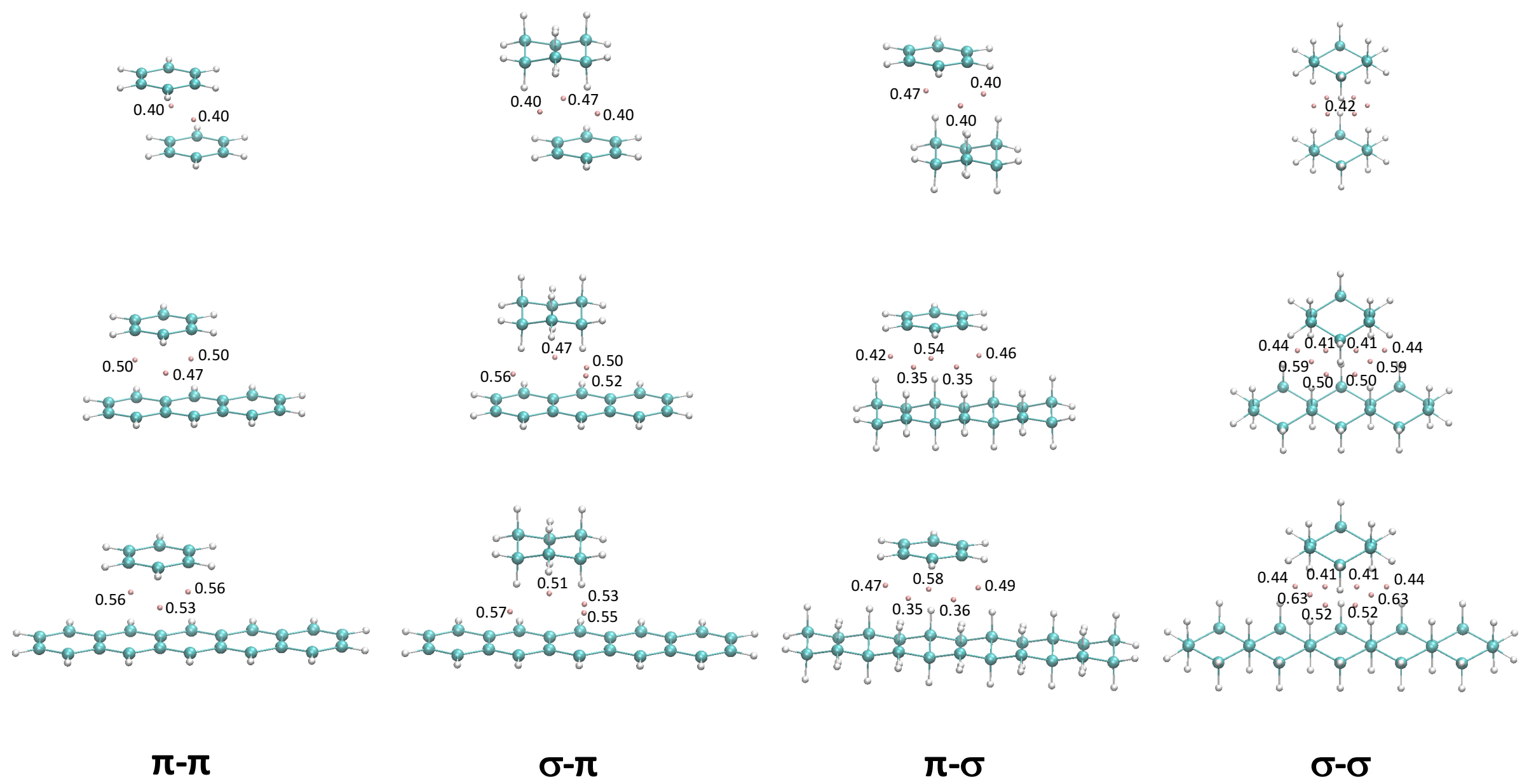

Figure 3. Values of the density at the intermolecular bond critical points of the dimers studied. The density (a.u.) is multiplied by a factor of 100. 

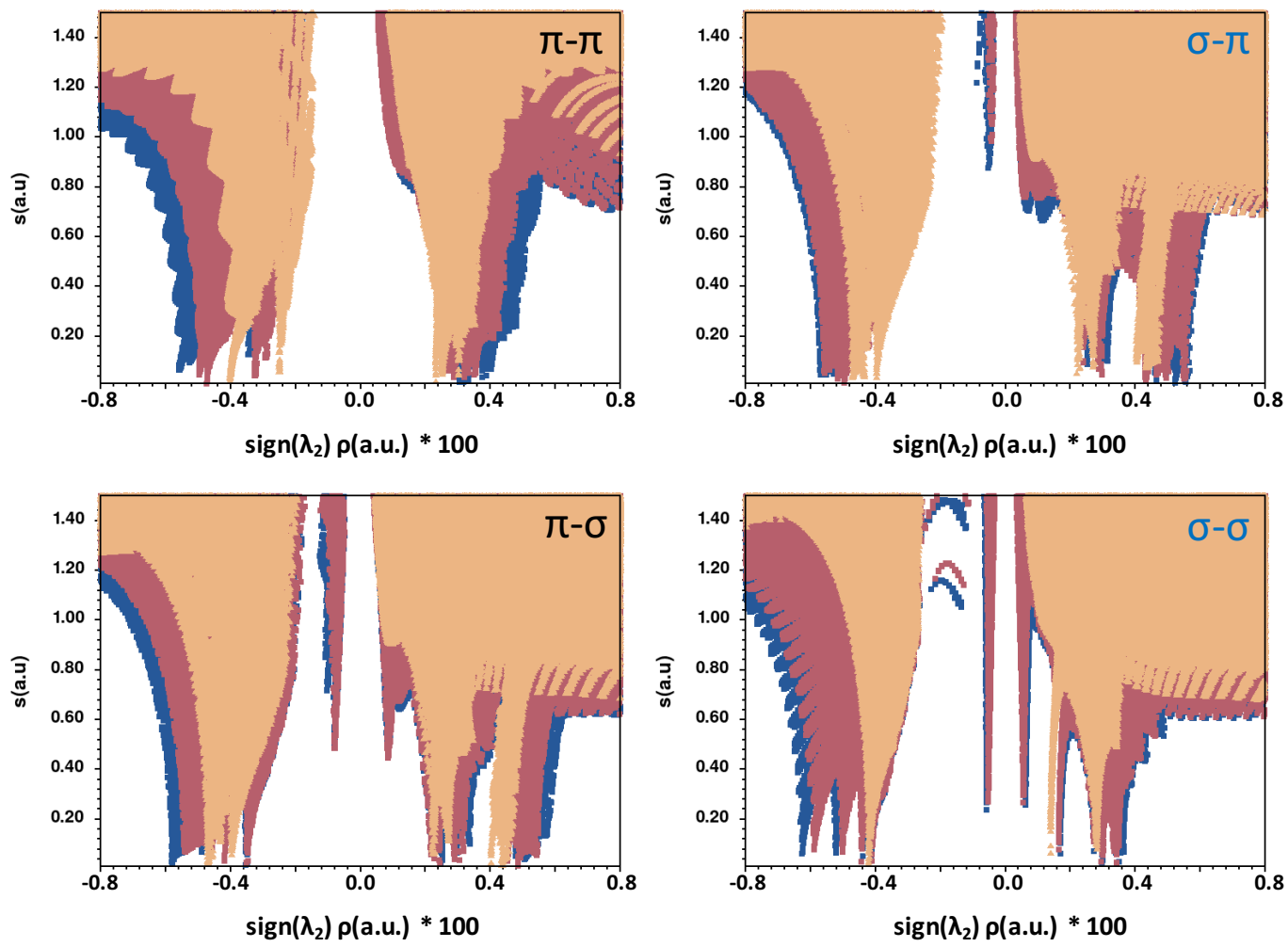

Figure 4. $\mathrm{NCl} 2 \mathrm{D}$ plots showing the interactions in the minima of the dimers studied.

The characteristics of the interaction as described with $\mathrm{NCl}$ can be visualized more quantitatively in Figure 4. The two-dimensional $\mathrm{NCl}$ plots reveal the interactions as peaks with near zero reduced density gradient. The plots in Figure 4 allow to follow the changes in these peaks and therefore in the interaction as the systems grow. These peaks can be related to the values of the density at the intermolecular bond critical points (BCPS) as revealed by QTAIM (Figure 3).

Considering $\pi-\pi$ complexes, benzene dimer shows a stabilising peak at density 0.004 a.u., corresponding to two equivalent $\mathrm{NCl}$ surfaces and BCPs. Benzene-anthracene complex shows peaks clearly displaced towards larger densities suggesting an increase in the stability of the complex. In the case of the complex with pentacene, the trend continues and the peaks displace towards larger densities, reaching values near 0.0056 a.u.. Therefore, in $\pi-\pi$ complexes, the densities at the BCPs increase noticeably as the size of the system grows, leading to more stable complexes. In $\sigma-\sigma$ complexes, the behaviour is somewhat more complex. There is a clear peak in cyclohexane dimer at density 0.0042 a.u., a magnitude similar to that in benzene dimer. The complex with $\mathrm{C}_{14}$ shows peaks displaced towards larger densities, suggesting an increase in the interaction. However, the increase of density when passing to $C_{22}$ is much smaller than the one observed in $\pi-\pi$ complexes. $\pi-\sigma$ complexes behave similarly, though the density decreases for several BCPs. In $\sigma-\pi$ complexes all BCPs increase their density as the system grows. There seems that the presence of extended $\pi$ systems allows for a more effective reinforcement of the interaction, probably also related to the shortening of equilibrium distances. 

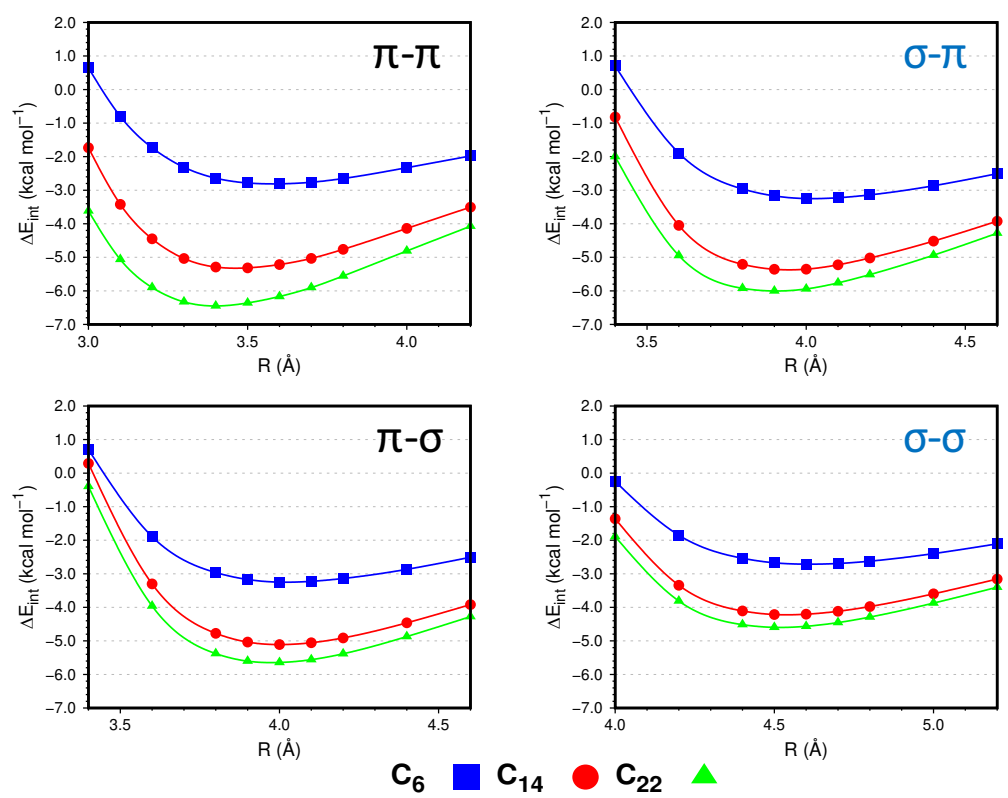

Figure 5. Potential energy curves obtained for the dimers considered. TPSS-D3BJ/def2TZVPP.

Figure 5 shows the potential energy curves obtained for the dimers considered as obtained at the TPSS-D3BJ/def2-TZVPP level, while the characteristics of the minima are listed in Table 1. Figure 5 clearly shows how the interaction increases as the size of the dimers grow. It is evident from Figure 5 and data in Table 1 that as the system size increases so it does the stability of the dimers. However, the rate for this increase is different depending on the nature of the systems. The stability of the dimers increase more quickly with size in $\pi-\pi$ complexes, while in aliphatic ones is almost converged in the $\mathrm{C}_{22}$ derivative. The results indicate that there is a significant stabilisation for all complexes when going from $C_{6}$ to $C_{14}$, because the contact surface between the species forming the dimer increases, leading to more favourable dispersion interactions. The stability of the complexes increase by $-2.5 \mathrm{kcal} \mathrm{mol}^{-1}$ in $\pi-\pi$ complexes while the increment is only of $-1.5 \mathrm{kcal} \mathrm{mol}^{-1}$ in $\sigma-\sigma$ ones. As a result, $\pi$-containing complexes are similarly stable with energy differences around $0.25 \mathrm{kcal} \mathrm{mol}^{-1}$ at most, while $\sigma-\sigma$ ones already become less favourable by around $1 \mathrm{kcal} \mathrm{mol}^{-1}$.

Going to $C_{22}$ dimers, $\pi-\pi$ complexes are further stabilised by $-1.1 \mathrm{kcal} \mathrm{mol}^{-1}$, while $\sigma-\sigma$ ones just increase by $-0.4 \mathrm{kcal} \mathrm{mol}^{-1}$. It is worth noticing that in mixed complexes, the extension of the aromatic system in $\sigma-\pi$ dimers allows a larger stability gain that when the $\sigma$ system is extended in $\pi-\sigma$ dimers. Though the differences are not large, $\sigma-\pi$ dimers already become around $-0.5 \mathrm{kcal}$ $\mathrm{mol}^{-1}$ more stable than $\pi-\sigma$ ones. Thus, the nature of the delocalised $\pi$ systems allows for a larger reinforcement of the interactions not observed in their aliphatic counterparts. This is also related to the shortening on equilibrium distances as observed in Table 1, leading to stronger interactions in extended $\pi$ systems. 
Table 1. Characteristics of the minima of the complexes studied. Equilibrium distances in $\AA$ and energies in $\mathrm{kcal} \mathrm{mol}^{-1}$. TPSS-D3BJ/def2-TZVPP.

\begin{tabular}{|l|l|l|l|l|l|l|c|}
\hline & & $\mathbf{R}_{\min }$ & $\Delta \mathbf{E}$ & & & $\mathbf{R}_{\min }$ & $\Delta \mathbf{E}$ \\
\hline $\boldsymbol{\pi}-\boldsymbol{\pi}$ & $\mathrm{C}_{6}$ & 3.58 & -2.81 & $\boldsymbol{\sigma}-\boldsymbol{\pi}$ & $\mathrm{C}_{6}$ & 4.02 & -3.25 \\
\hline & $\mathrm{C}_{14}$ & 3.46 & -5.32 & & $\mathrm{C}_{14}$ & 3.95 & -5.37 \\
\hline & $\mathrm{C}_{22}$ & 3.40 & -6.45 & & $\mathrm{C}_{22}$ & 3.91 & -6.00 \\
\hline $\boldsymbol{\pi}-\boldsymbol{\sigma}$ & $\mathrm{C}_{6}$ & 4.02 & -3.25 & $\sigma-\sigma$ & $\mathrm{C}_{6}$ & 4.61 & -2.71 \\
\hline & $\mathrm{C}_{14}$ & 4.00 & -5.11 & & $\mathrm{C}_{14}$ & 4.54 & -4.22 \\
\hline & $\mathrm{C}_{22}$ & 3.97 & -5.65 & & $\mathrm{C}_{22}$ & 4.52 & -4.60 \\
\hline
\end{tabular}

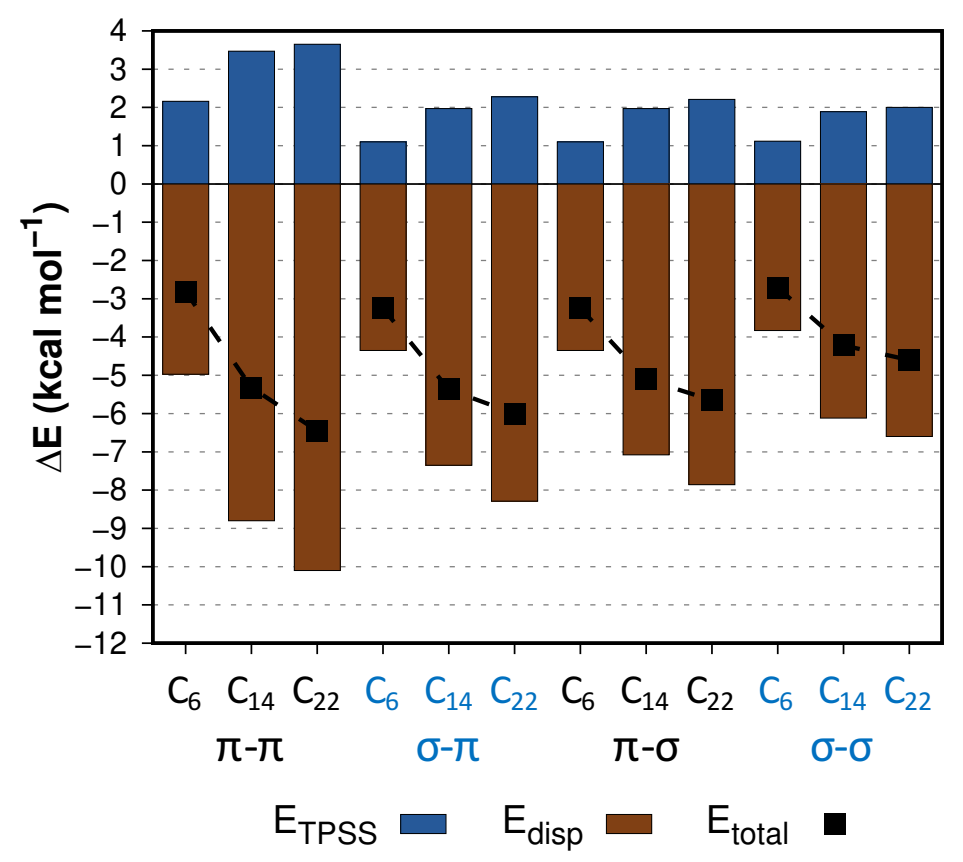

Figure 6. Energy components at the minima of the dimers.

Figure 6 decomposes the interaction energy into the contributions coming from the TPSS functional and the empirical dispersion term. It can be observed that, as expected, dispersion increases as the size of the system grows, but it does so much faster in $\pi-\pi$ complexes than in $\sigma-\sigma$ ones. Also, it can be appreciated in Figure 6 that in $\sigma-\pi$ complexes dispersion grows faster than in $\pi-\sigma$ ones, overcoming the increases in the repulsive TPSS component and leading to more stable complexes. The increments in the repulsive TPSS contribution are also related to the shortening of the equilibrium distances, so they change the most for $\pi-\pi$ and $\sigma-\pi$ complexes. In any case, dispersion contribution increases even more as the distance shortens so the stability of the complexes increases. 


\section{References}

1. Johnson, D. W.; Hof, F., Aromatic Interactions: Frontiers in Knowledge and Application. The Royal Society of Chemistry: 2017.

2. Tsuzuki, S.; Uchimaru, T., Magnitude and physical origin of intermolecular interactions of aromatic molecules: recent progress of computational studies. Curr. Org. Chem. 2006, 10, (7), 745-762. Doi: $10.2174 / 138527206776818937$.

3. Martinez, C. R.; Iverson, B. L., Rethinking the term "pi-stacking". Chemical Science 2012, 3, (7), 2191-2201. Doi: 10.1039/C2SC20045G.

4. Grimme, S., Do Special Noncovalent $\pi-\pi$ Stacking Interactions Really Exist? Angew. Chem. Int. Ed. 2008, 47, (18), 3430-3434. Doi: 10.1002/anie.200705157.

5. Cabaleiro-Lago, E. M.; Rodríguez-Otero, J., $\sigma-\sigma, \sigma-\pi$, and $\pi-\pi$ Stacking Interactions between SixMembered Cyclic Systems. Dispersion Dominates and Electrostatics Commands. ChemistrySelect 2017, 2, 5157-5166. Doi: 10.1002/slct.201700671.

6. Ninkovic, D. B.; Vojislavljevic-Vasilev, D. Z.; Medakovic, V. B.; Hall, M. B.; Brothers, E. N.; Zaric, S. D., Aliphatic-aromatic stacking interactions in cyclohexane-benzene are stronger than aromaticaromatic interaction in the benzene dimer. Phys: Chem. Chem. Phys. 2016, 18, (37), 25791-25795. Doi: $10.1039 / \mathrm{C} 6 \mathrm{CP} 03734 \mathrm{H}$.

7. Ehrlich, S.; Moellmann, J.; Grimme, S., Dispersion-Corrected Density Functional Theory for Aromatic Interactions in Complex Systems. Acc. Chem. Res. 2013, 46, 916-926. Doi: 10.1021/Ar3000844.

8. Cabaleiro-Lago, E. M.; Rodríguez-Otero, J., On the Nature of $\sigma-\sigma, \sigma-\pi$, and $\pi-\pi$ Stacking in Extended Systems. ACS Omega 2018, 3, (8), 9348-9359. Doi: 10.1021/acsomega.8b01339.

9. TURBOMOLE V6.3 2011, a development of University of Karlsruhe and Forschungszentrum Karlsruhe GmbH, 1989-2007, TURBOMOLE GmbH, since 2007; available from http://www.turbomole.com/.

10. Contreras-García, J.; Johnson, E. R.; Keinan, S.; Chaudret, R.; Piquemal, J.-P.; Beratan, D. N.; Yang, W., NCIPLOT: A Program for Plotting Noncovalent Interaction Regions. J. Chem. Theory Comput. 2011, 7, (3), 625-632. Doi: 10.1021/ct100641a.

11. Johnson, E. R.; Keinan, S.; Mori-Sánchez, P.; Contreras-García, J.; Cohen, A. J.; Yang, W., Revealing Noncovalent Interactions. J. Am. Chem. Soc. 2010, 132, (18), 6498-6506. Doi: 10.1021/ja100936w. 